\title{
RESTRICTED 132-ALTERNATING PERMUTATIONS AND CHEBYSHEV POLYNOMIALS
}

\author{
Toufik Mansour ${ }^{1}$ \\ Department of Mathematics, Chalmers University of Technology, S-41296 Göteborg, Sweden \\ toufik@math.chalmers.se
}

ABSTRACT

A permutation is said to be alternating if it starts with rise and then descents and rises come in turn. In this paper we study the generating function for the number of alternating permutations on $n$ letters that avoid or contain exactly once 132 and also avoid or contain exactly once an arbitrary pattern on $k$ letters. In several interesting cases the generating function depends only on $k$ and is expressed via Chebyshev polynomials of the second kind.

2000 Mathematics Subject Classification: Primary 05A05, 05A15; Secondary 30B70, 42C05

\section{INTRODUCTION}

The aim of this paper is to give analogues of enumerative results on certain classes of permutations characterized by pattern-avoidance in the symmetric group $\mathfrak{S}_{n}$. In the set of alternating permutations we identify classes of restricted alternating permutations with enumerative properties analogous to results on permutations. More precisely, we study generating functions for the number of alternating permutations that avoid or contain exactly once 132 and also avoid or contain exactly once an arbitrary permutation $\tau \in \mathfrak{S}_{k}$. In the remainder of this section, we present a brief account of earlier works which motivated our investigation, we give the basic definitions used throughout the paper, and the organization of this paper.

1.1. Classical patterns. Let $\alpha \in \mathfrak{S}_{n}$ and $\tau \in \mathfrak{S}_{k}$ be two permutations. We say that $\alpha$ contains $\tau$ if there exists a subsequence $1 \leq i_{1}<i_{2}<\cdots<i_{k} \leq n$ such that $\left(\alpha_{i_{1}}, \ldots, \alpha_{i_{k}}\right)$ is order-isomorphic to $\tau$; in such a context $\tau$ is usually called a pattern. We say that $\alpha$ avoids $\tau$, or is $\tau$-avoiding, if such a subsequence does not exist. The set of all $\tau$-avoiding permutations in $\mathfrak{S}_{n}$ is denoted $\mathfrak{S}_{n}(\tau)$. For an arbitrary finite collection of patterns $T$, we say that $\alpha$ avoids $T$ if $\alpha$ avoids any $\tau \in T$; the corresponding subset of $\mathfrak{S}_{n}$ is denoted $\mathfrak{S}_{n}(T)$.

While the case of permutations avoiding a single pattern has attracted much attention, the case of multiple pattern avoidance remains less investigated. In particular, it is natural, as the next step, to consider permutations avoiding pairs of patterns $\tau_{1}, \tau_{2}$. This problem was solved completely for $\tau_{1}, \tau_{2} \in \mathfrak{S}_{3}$ (see $[\mathrm{SS}]$ ), for $\tau_{1} \in \mathfrak{S}_{3}$ and $\tau_{2} \in \mathfrak{S}_{4}$ (see $[\mathrm{W}]$ ), and for $\tau_{1}, \tau_{2} \in \mathfrak{S}_{4}$ (see [Bo, Km] and references therein). Several recent papers [CW, MV1, Kr, MV2, MV3, MV4] deal with the case

\footnotetext{
${ }^{1}$ Research financed by EC's IHRP Programme, within the Research Training Network "Algebraic Combinatorics in Europe", grant HPRN-CT-2001-00272
} 
$\tau_{1} \in \mathfrak{S}_{3}, \tau_{2} \in \mathfrak{S}_{k}$ for various pairs $\tau_{1}, \tau_{2}$. Another natural question is to study permutations avoiding $\tau_{1}$ and containing $\tau_{2}$ exactly $t$ times. Such a problem for certain $\tau_{1}, \tau_{2} \in \mathfrak{S}_{3}$ and $t=1$ was investigated in $[R]$, and for certain $\tau_{1} \in \mathfrak{S}_{3}, \tau_{2} \in \mathfrak{S}_{k}$ in [RWZ, MV1, Kr]. The tools involved in these papers include Fibonacci numbers, Lucas numbers, Catalan numbers, Chebyshev polynomials, continued fractions, and Dyck words.

Fibonacci numbers are defined by $F_{0}=0, F_{1}=1$, and $F_{n}=F_{n-1}+F_{n-2}$ for all $n \geq 2$.

Lucas numbers are defined by $L_{0}=2, L_{1}=1$, and $L_{n}=L_{n-1}+L_{n-2}$ for all $n \geq 2$.

Catalan numbers are defined by $C_{n}=\frac{1}{n+1}\left(\begin{array}{c}2 n \\ n\end{array}\right)$ for all $n \geq 0$. The generating function for the Catalan numbers is given by $C(x)=\frac{1-\sqrt{1-4 x}}{2 x}$.

Chebyshev polynomials of the second kind (in what follows just Chebyshev polynomials) are defined by $U_{r}(\cos \theta)=\frac{\sin (r+1) \theta}{\sin \theta}$ for $r \geq 0$. Evidently, $U_{r}(t)$ is a polynomial of degree $r$ in $t$ with integer coefficients, and satisfy

$$
U_{0}(t)=1, U_{1}(t)=2 t \text {, and } U_{r}(t)=2 t U_{r-1}(t)-U_{r-2}(t) \text { for all } r \geq 2 .
$$

Chebyshev polynomials were invented for the needs of approximation theory, but are also widely used in various other branches of mathematics, including algebra, combinatorics, and number theory (see $[\mathrm{Ri}]$ ). Apparently, for the first time the relation between restricted permutations and Chebyshev polynomials was discovered by Chow and West in [CW], and later by Mansour and Vainshtein [MV1, MV2, MV3, MV4], Krattenthaler [Kr]. These results related to a rational function

$$
R_{k}(x)=\frac{U_{k-1}\left(\frac{1}{2 \sqrt{x}}\right)}{\sqrt{x} U_{k}\left(\frac{1}{2 \sqrt{x}}\right)},
$$

for all $k \geq 1$. It is easy to see that for any $k, R_{k}(x)$ is rational in $x$ and satisfies

$$
R_{k}(x)=\frac{1}{1-x R_{k-1}(x)},
$$

for all $k \geq 1$.

1.2. Generalized patterns. In [BS], there were introduced generalized permutation patterns that allow the requirement that two adjacent letters in a pattern must be adjacent in the permutation. We write a classical pattern with dashes between any two adjacent letters of the pattern, say 1342, as 1-3-4-2, and if we write, say 24-3-1, then we mean that if this pattern occurs in a permutation $\pi$, then the letters in $\pi$ that correspond to 2 and 4 are adjacent (for more details, see $[\mathrm{C}]$ ). For example, the permutation $\pi=35421$ has only two occurrences of the pattern 23-1, namely the subsequences 352 and 351, whereas $\pi$ has four occurrences of the pattern 2-3-1, namely the subsequences $352,351,342$ and 341. In [MV1, M1, M2, M3], there were studied the number of permutations that avoid or contain exactly once 1-3-2 and that also avoid or contain exactly once an arbitrary generalized pattern. The tools involved in these papers include Fibonacci numbers, Catalan numbers, Chebyshev polynomials, and continued fractions.

1.3. Alternating permutations. A permutation is said to be alternating if it starts with rise and then descents and rises come in turn. In other words, an alternating permutation $\pi \in \mathfrak{S}_{n}$ satisfies $\pi_{2 j-1}<\pi_{2 j}>\pi_{2 j+1}$ for all $1 \leq j \leq[n / 2]$, that is to say its rise (resp. descent) is equal to an odd (resp. even) index. We denote the set of all alternating permutations on $n$ letters by $A_{n}$. Other names 
that authors have used for these permutations are zig-sag permutation and up down permutations. The determination of the number of alternating permutations for the set $\{1,2, \ldots, n\}$ (or on $n$ letters) is known as André's problem (see [A1, A2]). An example of an alternating permutation is 14253. The number of alternating permutations on $n$ letters, for $n=1,2, \ldots, 10$, is $1,1,2,5,16,61,272,1385$, 7936, 50521 (see [SP, Sequence A000111(M1492)]). These numbers are known as the Euler numbers and have exponential generating function $\sec x+\tan x$.

A permutation is said to be up-up (resp. up-down, down-up, down-down) if it starts with rise (resp. rise, descent, descent), then descents and rises (resp. descents and rises, rises and descents, rises and descents) come in turn, and ends with rise (resp. descent, rise, descent). We denote the set of all up-up (resp. up-down, down-up, down-down) permutations of length $n$ by $U U_{n}$ (resp. $U D_{n}, D U_{n}$, $D D_{n}$ ) for all $n \geq 2$ (for $n=0,1$ we define $U U_{n}=U D_{n}=D U_{n}=D D_{n}=\varnothing$ ). Clearly, for all $n \geq 2$,

$$
A_{n}=U U_{n} \cup U D_{n} .
$$

1.4. Organization of the paper. In this paper we use generating function techniques to study those alternating (up-up, up-down, down-up, down-down) permutations that avoid or contain exactly once 1-3-2 and that also avoid or contain exactly once an arbitrary generalized pattern on $k$ letters.

The paper is organized as follows. The case of alternating (up-up, up-down, down-up, down-down) permutations that avoid both 1-3-2 and an arbitrary generalized pattern $\tau$ is treated in Section 2 . We derive a simple structure for alternating (up-up, up-down, down-up, down-down) permutations that avoid 1-3-2. This structure can be used for several interesting cases, including the classical pattern $\tau=1-2-\cdots-k$ and $\tau=2-3-\cdots-k-1$, the generalized patterns $\tau=12-3-\cdots-k$ and $\tau=21-3-\cdots-k$. The case of alternating (up-up, up-down, down-up, down-down) permutations that avoid 1-3-2 and contain exactly once $\tau$ is treated in Section 3. Here again, we use the structure of alternating (up-up, up-down, down-up, down-down) permutations that avoid 1-3-2 for several particular cases. The case of alternating (up-up, up-down, down-up, down-down) permutations that contain 1-3-2 exactly once is treated in Sections 4 and 5 for avoiding $\tau$, or containing $\tau$ exactly once, respectively. Finally, in Section 6 we present several directions to extend and generalize the results of the previous sections, including a statistics on the set of alternating (up-up, up-down, down-up, down-down) permutations that avoid 1-3-2.

Most of the explicit solutions obtained in Sections 2-5 involve Chebyshev polynomials of the second kind.

\section{AVOIDING 1-3-2 AND ANOTHER PATTERN $\tau$}

In this section we consider those alternating (up-up, up-down, down-up, down-down) permutations in $\mathfrak{S}_{n}$ that avoid 1-3-2 and avoid another generalized pattern $\tau$. We begin by setting some notation. Let $a_{\tau}(n)$ denote the number of alternating permutations in $A_{n}(1-3-2, \tau)$, and let $A_{\tau}(x)=\sum_{n \geq 0} A_{\tau}(n) x^{n}$ be the corresponding generating function.

Moreover, we define $u d_{\tau}(n), u u_{\tau}(n), d u_{\tau}(n)$, and $d d_{\tau}(n)$ as the number of up-up, up-down, down-up, and down-down permutation in $\mathfrak{S}_{n}(1-3-2, \tau)$, respectively. We denote, the corresponding generating functions by $U D_{\tau}(x), U U_{\tau}(x), D U_{\tau}(x)$, and $D D_{\tau}(x)$, respectively. In this section we describe a method for enumerating alternating (up-up, up-down, down-up, down-down) permutations that avoid 1-3-2 and another generalized pattern $\tau$ and we use out this method to enumerate $a_{\tau}(n)$ for various $\tau$. 
FigurE 1. The block decomposition for $\alpha \in \mathfrak{S}_{n}(1-3-2)$

We begin with an observation concerning the structure of the alternating (up-up, up-down, down-up, down-down) permutations that avoid 1-3-2.

Let $\alpha \in \mathfrak{S}_{n}$ (1-3-2); by using the block decomposition approach (see [MV4]) we have one possibility for block decomposition, as described in Figure 1, if $\alpha=\left(\alpha^{\prime}, n, \alpha^{\prime \prime}\right)$, then every entry of $\alpha^{\prime}$ is greater than every entry of $\alpha^{\prime \prime}$. Therefore, by using the block decomposition of $\alpha$, we get the following proposition which is the base for all the results in this section.

Proposition 2.1. Let $\alpha=\left(\alpha^{\prime}, n, \alpha^{\prime \prime}\right) \in \mathfrak{S}_{n}(1-3-2)$.

(1) If $\alpha \in U D_{n}$ (1-3-2), then the block decomposition of $\alpha$ can have one of the following possibilities:

(1.1) $\alpha^{\prime}$ is an 1-3-2-avoiding up-down permutation on the letters $n-j+1, n-j+2, \ldots, n-1$, and either $\alpha^{\prime \prime}$ is an 1-3-2-avoiding up-down permutation on the letters $1,2, \ldots, n-j$ or $\alpha^{\prime \prime}=1$;

(1.2) $\alpha^{\prime}=n-1$ and either $\alpha^{\prime \prime}$ is an 1-3-2-avoiding up-down permutation on the letters $1,2, \ldots, n-j-1$ or $\alpha^{\prime \prime}=1$.

(2) If $\alpha \in U U_{n}$ (1-3-2), then the block decomposition of $\alpha$ can have one of the following possibilities:

(2.1) $\alpha^{\prime}=n-1$ and either $\alpha^{\prime \prime}=\varnothing$ or $\alpha^{\prime \prime}$ is an 1-3-2-avoiding up-up permutation on the letters $1,2, \ldots, n-2$

(2.2) $\alpha^{\prime \prime}=\varnothing$ and $\alpha^{\prime}$ is an 1-3-2-avoiding up-down permutation on the letters $1,2, \ldots, n-1$;

(2.3) $\alpha^{\prime}$ is an 1-3-2-avoiding up-down permutation on the letters $n-j+1, \ldots, n-2, n-1$, and $\alpha^{\prime \prime}$ is an 1-3-2-avoiding up-up permutation on the letters $1,2, \ldots, n-j$.

(3) If $\alpha \in D U_{n}$ (1-3-2), then the block decomposition of $\alpha$ can have one of the following possibilities:

(3.1) $\alpha^{\prime}=\varnothing$, and $\alpha^{\prime \prime}$ is an 1-3-2-avoiding up-up permutation on the letters $1,2, \ldots, n-1$;

(3.2) $\alpha^{\prime}$ is an 1-3-2-avoiding down-down permutation on the letters $n-j+1, \ldots, n-2, n-1$, and either $\alpha^{\prime \prime}=\varnothing$ or $\alpha^{\prime \prime}$ is an 1-3-2-avoiding up-up permutation on the letters $1,2, \ldots, n-j$.

(4) If $\alpha \in D D_{n}(1-3-2)$, then the block decomposition of $\alpha$ can have one of the following possibilities:

(4.1) $\alpha^{\prime}=\varnothing$, and $\alpha^{\prime \prime}$ is an 1-3-2-avoiding up-down permutation on the letters $1,2, \ldots, n-1$;

(4.2) $\alpha^{\prime}$ is an 1-3-2-avoiding down-down permutation on the letters $n-j+1, \ldots, n-2, n-1$, and either $\alpha^{\prime \prime}=1$ or $\alpha^{\prime \prime}$ is an 1-3-2-avoiding up-down permutation on the letters $1,2, \ldots, n-j$.

2.1. The pattern $\tau=\varnothing$. Using Proposition 2.1, we now enumerate those alternating (up-up, updown, down-up, down-down) permutations in $\mathfrak{S}_{n}$ that avoid 1-3-2.

Theorem 2.2. The number of alternating permutations of length $n$ that avoid 1-3-2 is given by $C_{[n / 2]}$ for any $n \geq 0$. Moreover, for all $n \geq 3$,

(1) the number of up-down permutations of length $n$ that avoid 1-3-2 is given by $C_{(n-1) / 2}$, 
(2) the number of up-up permutations of length $n$ that avoid 1-3-2 is given by $C_{n / 2}$,

(3) the number of down-up permutation of length $n$ that avoid 1-3-2 is given by $C_{(n+1) / 2}$,

(4) the number of down-down permutations of length $n$ that avoid 1-3-2 is given by $C_{n / 2}$,

where $C_{m}$ is the mth Catalan number, such that $C_{m}$ is assumed to be 0 whenever $m$ is a non-integer number.

Proof. An alternating permutation can be either up-down, or up-up, or down-up, or down-down. If we write an equation for every case in terms of generating functions and use Proposition 2.1, then we get

$$
\left\{\begin{array}{l}
U D_{\varnothing}(x)=x\left(U D_{\varnothing}(x)+x\right)^{2} \\
D U_{\varnothing}(x)=x U U_{\varnothing}(x)+x D D_{\varnothing}(x) U U_{\varnothing}(x)+x D D_{\varnothing}(x), \\
D D_{\varnothing}(x)=x\left(x+U D_{\varnothing}(x)\right)+x D D_{\varnothing}(x)\left(x+U D_{\varnothing}(x)\right) \\
U U_{\varnothing}(x)=x^{2}\left(U U_{\varnothing}(x)+1\right)+x U D_{\varnothing}(x)+x U D_{\varnothing}(x) U U_{\varnothing}(x) .
\end{array}\right.
$$

Therefore, solving the above equations, we get

$$
\begin{array}{lll}
U D_{\varnothing}(x)=\frac{1-2 x^{2}-\sqrt{1-4 x^{2}}}{2 x}, & D U_{\varnothing}(x)=x\left(\frac{1-\sqrt{1-4 x^{2}}}{2 x^{2}}\right)^{2}-x, \\
D D_{\varnothing}(x)=\frac{1-\sqrt{1-4 x^{2}}}{1+\sqrt{1-4 x^{2}}}, & & U U_{\varnothing}(x)=\frac{1-\sqrt{1-4 x^{2}}}{1+\sqrt{1-4 x^{2}}} .
\end{array}
$$

Hence, the rest is easy to check by using $A_{\varnothing}(x)=1+x+U D_{\varnothing}(x)+U U_{\varnothing}(x)$ (see Equation 1.4).

Using Proposition 2.1, we now enumerate various sets of alternating (up-up, up-down, down-up, down-down) permutations in $\mathfrak{S}_{n}$ that avoid 1-3-2 and nonempty generalized pattern $\tau$.

2.2. A classical pattern $\tau=1-2-\cdots-k$. Let us start by the following example.

Example 2.3. By definitions we have that $U D_{1}(x)=D D_{1}(x)=U U_{1}(x)=D U_{1}(x)=0, U D_{1-2}(x)=$ $U U_{1-2}(x)=D U_{1-2}(x)=0$, and $D D_{1-2}(x)=x^{2}$.

The case of varying $k$ is more interesting. As an extension of Example 2.3, let us consider the case $\tau=1-2-\cdots-k$.

Theorem 2.4. For all $k \geq 2$,

(1) $U D_{1-2-\cdots-k}(x)=\frac{x U_{k-3}\left(\frac{1}{2 x}\right)}{U_{k-1}\left(\frac{1}{2 x}\right)}$;

(2) $D D_{1-2-\cdots-k}(x)=\frac{x^{k-1}+U_{k-3}\left(\frac{1}{2 x}\right)}{U_{k-1}\left(\frac{1}{2 x}\right)}$;

(3) $U U_{1-2-\cdots-k}(x)=\frac{U_{k-3}\left(\frac{1}{2 x}\right)}{U_{k-1}\left(\frac{1}{2 x}\right)}$;

(4) $D U_{1-2-\cdots-k}(x)=\frac{x^{k-1}+U_{k-3}\left(\frac{1}{2 x}\right)}{x U_{k-1}\left(\frac{1}{2 x}\right)}-x$;

where $U_{-1}(t)=0$.

Proof. Let us verify (1). By using Proposition 2.1, we have that

$$
U D_{1-2-\cdots-k}(x)=x\left(U D_{1-2-\cdots-k}(x)+x\right)\left(U D_{1-2-\cdots-(k-1)}(x)+x\right),
$$


equivalently,

$$
U D_{1-2-\cdots-k}(x)=\frac{x^{2}\left(x+U D_{1-2-\cdots-(k-1)}(x)\right)}{1-x^{2}-x U D_{1-2-\cdots-(k-1)}(x)} .
$$

Now, assume that (1) holds for $k-1$. So, by Identity 1.2 we get that

$$
U D_{1-2-\cdots-(k-1)}(x)=x^{3} R_{k-3}\left(x^{2}\right) R_{k-2}\left(x^{2}\right) .
$$

Therefore,

$$
U D_{1-2-\cdots-k}(x)=\frac{x^{2}\left(x+x^{3} R_{k-3}\left(x^{2}\right) R_{k-2}\left(x^{2}\right)\right)}{1-x^{2}-x^{4} R_{k-3}\left(x^{2}\right) R_{k-2}\left(x^{2}\right)},
$$

and using Identity 1.3, we get

$$
U D_{1-2-\cdots-k}(x)=x^{3} R_{k-2}\left(x^{2}\right) R_{k-1}\left(x^{2}\right) .
$$

So, by Equation 1.2, (1) holds for $k$. Hence, by induction on $k$ together with Example 2.3 we get the desired result as claimed in (1).

Let us verify (2). By using Proposition 2.1, we get

$$
D D_{1-2-\cdots-k}(x)=x\left(x+U D_{1-2-\cdots-k}(x)\right)\left(1+D D_{1-2-\cdots-(k-1)}(x)\right) .
$$

Using (1), Equation 1.3, and induction on $k$ together with Example 2.3 we get the desired result as claimed in (2).

The rest is easy to check by using the fact that $U D_{1-2-\cdots-k}(x)=x U U_{1-2-\cdots-k}(x)$ and $D D_{1-2-\cdots-k}(x)=$ $x D U_{1-2-\cdots-k}(x)+x^{2}$.

As a corollary to Theorem 2.4 and Equation 1.4, we get the number of alternating permutations in $A_{n}(1-3-2,1-2-\cdots-k)$.

Corollary 2.5. For all $k \geq 2$,

$$
A_{1-2-\cdots-k}(x)=\frac{(1+x) U_{k-2}\left(\frac{1}{2 x}\right)}{x U_{k-1}\left(\frac{1}{2 x}\right)}=(1+x) R_{k-1}\left(x^{2}\right) .
$$

Corollary 2.5 , for $k=5$, yields that the number of alternating permutations in $A_{n}(1-3-2,1-2-3-4-5)$ is given by $F_{2[n / 2]-1}$, where $F_{m}$ is the $m$ th Fibonacci number.

2.3. A classical pattern $\tau=2-3-\cdots-k-1$. By Proposition 2.1, we have that $U D_{2-3-1}(x)=0$ and $D D_{2-3-1}(x)=x^{2}$. The case of varying $k$ is more interesting. As an extension of this example, let us consider the case $\tau=2-3-\cdots-k-1$.

Theorem 2.6. For all $k \geq 3$,

(1) $U D_{2-3-\cdots-k-1}(x)=\frac{x U_{k-4}\left(\frac{1}{2 x}\right)}{U_{k-2}\left(\frac{1}{2 x}\right)}$,

(2) $D D_{2-3-\cdots-k-1}(x)=\frac{x^{k-2}+U_{k-4}\left(\frac{1}{2 x}\right)}{U_{k-2}\left(\frac{1}{2 x}\right)}$,

(3) $U U_{2-3-\cdots-k-1}(x)=\frac{U_{k-3}^{2}\left(\frac{1}{2 x}\right)}{U_{k-2}^{2}\left(\frac{1}{2 x}\right)}$,

(4) $D U_{2-3-\cdots-k-1}(x)=\frac{x}{U_{k-2}^{2}\left(\frac{1}{2 x}\right)}\left(x^{k-3}\left(U_{k-3}\left(\frac{1}{2 x}\right)+x U_{k-2}\left(\frac{1}{2 x}\right)\right)+2 U_{k-3}^{2}\left(\frac{1}{2 x}\right)+U_{k-4}^{2}\left(\frac{1}{2 x}\right)-2\right)$. 
Proof. Proposition 2.1(1) yields

$$
U D_{2-3-\cdots-k-1}(x)=x\left(U D_{2-3-\cdots-k-1}(x)+x\right)\left(U D_{1-2-\cdots-(k-2)}(x)+x\right) .
$$

Using Equation 1.3 and Recurrence 1.1, together with Theorem 2.4(1), we have that case (1) holds.

Proposition 2.1(4) yields

$$
D D_{2-3-\cdots-k-1}(x)=x\left(x+U D_{2-3-\cdots-k-1}(x)\right)\left(1+U D_{1-2-\cdots-(k-2)}(x)\right) .
$$

By using the first case (1) and Theorem 2.4(2), together with Equation 1.3 and Recurrence 1.1, we get that case (2) holds.

Proposition 2.1(2) yields

$$
\left.U U_{2-3-\cdots-k-1}(x)=x^{2}\left(1+U U_{2-3-\cdots-k-1}(x)\right)+x U D_{2-3-\cdots-k-1}(x)+x U D_{1-2-\cdots-(k-2)}(x) U U_{2-3-\cdots-k-1}(x)\right) \text {. }
$$

By using cases (1) and (2), together with Theorem 2.4, Equation 1.3, and Recurrence 1.1, we have that case (3) holds.

Proposition 2.1(3) yields

$$
D U_{2-3-\cdots-k-1}(x)=x U U_{2-3-\cdots-k-1}(x)+x D D_{1-2-\cdots-(k-2)}(x) U U_{2-3-\cdots-k-1}(x)+x D D_{2-3-\cdots-k-1}(x) .
$$

By using cases (3) and (4), Theorem 2.4(2) and Recurrence 1.1, we get that case (4) holds.

Theorem 2.6 yields an explicit formula to the generating function for the number of alternating permutations in $A_{n}(1-3-2,2-3-\cdots-k-1)$.

Corollary 2.7. For all $k \geq 3$,

$$
A_{2-3-\cdots-k-1}(x)=\frac{(1+x) U_{k-3}^{2}\left(\frac{1}{2 x}\right)-x}{U_{k-2}^{2}\left(\frac{1}{2 x}\right)} .
$$

For example, by using Corollary 2.7 we get that for all $n \geq 1$,

$$
A_{2-3-4-5-6-1}(2 n)=\frac{7}{10} n L_{2 n}-\frac{1}{10}(15 n-4) F_{2 n} \text { and } A_{2-3-4-5-6-1}(2 n+1)=F_{2 n-1},
$$

where $F_{m}$ is the $m$ th Fibonacci number and $L_{m}$ is the $m$ th Lucas number.

2.4. A generalized patterns $\tau-3-\cdots-k$. In the current subsection we interesting in alternating permutations with two restrictions, the first one is 1-3-2 (classical pattern 132) and the second one is $\tau-3-\cdots-k$ (generalized pattern), where $\tau=12, \tau=21, \tau=1-2$, or $\tau=2-1$. Using the same arguments as in the proof of Theorem 2.4, we get

Theorem 2.8. Let $\tau \in\{12,21,1-2,2-1\}$. For all $k \geq 2$,

(1) $U D_{\tau-3-\cdots-k}(x)=\frac{x U_{k-3}\left(\frac{1}{2 x}\right)}{U_{k-1}\left(\frac{1}{2 x}\right)}$,

(2) $D D_{\tau-3-\cdots-k}(x)=\frac{x^{k-1}+U_{k-3}\left(\frac{1}{2 x}\right)}{U_{k-1}\left(\frac{1}{2 x}\right)}$,

(3) $U U_{\tau-3-\cdots-k}(x)=\frac{U_{k-3}\left(\frac{1}{2 x}\right)}{U_{k-1}\left(\frac{1}{2 x}\right)}$,

(4) $D U_{\tau-3-\cdots-k}(x)=\frac{x^{k-1}+U_{k-3}\left(\frac{1}{2 x}\right)}{x U_{k-1}\left(\frac{1}{2 x}\right)}-x$. 
The above theorem together with Equation 1.4 yield that the number of alternating permutations in $A_{n}(1-3-2, \tau-3-\cdots-k)$, where $\tau \in\{12,21,1-2,2-1\}$.

Corollary 2.9. Let $\tau \in\{12,21,1-2,2-1\}$. For any $k \geq 2$,

$$
A_{\tau-3-\cdots-k}(x)=\frac{(1+x) U_{k-2}\left(\frac{1}{2 x}\right)}{x U_{k-1}\left(\frac{1}{2 x}\right)}=(1+x) R_{k-1}\left(x^{2}\right) .
$$

Corollary 2.9 suggests that there should exist a bijection between the sets $A_{n}(1-3-2,1-2-\cdots-k)$ and $A_{n}(1-3-2,2-1-3-\cdots-k)$. However, we failed to produce such a bijection, and finding it remains a challenging open question.

\section{Avoiding 1-3-2 AND CONTAINING ANOTHER PATtERN}

In this section we consider those alternating (up-up, up-down, down-up, down-down) permutations in $\mathfrak{S}_{n}$ that avoid 1-3-2 and contain another generalized pattern $\tau$ exactly once. We begin by setting some notation. Let $a_{\tau ; r}(n)$ be the number of alternating permutations in $A_{n}(1-3-2)$ that contain $\tau$ exactly $r$ times. Moreover, we denote the number of up-up (resp. up-down, down-up, down-down) permutations in $\mathfrak{S}_{n}(1-3-2)$ that contain $\tau$ exactly $r$ times by $u u_{\tau ; r}(n)\left(\operatorname{resp} . u d_{\tau ; r}(n), d u_{\tau ; r}(n), d d_{\tau ; r}(n)\right)$. We denote the corresponding generating function by $A_{\tau ; r}(x)$ (resp. $U U_{\tau ; r}(x), U D_{\tau ; r}(x), D U_{\tau ; r}(x), D D_{\tau ; r}(x)$ ). In this section we use out Proposition 2.1 and generating function techniques to enumerate $a_{\tau ; r}(n)$, $u d_{\tau ; r}(n), u u_{\tau ; r}(x), d u_{\tau ; r}(n)$, and $d d_{\tau ; r}(n)$ for various cases of $\tau$.

\subsection{A classical pattern $\tau=1-2-\cdots-k$.}

Theorem 3.1. For all $k \geq 2$,

(1) $U D_{1-2-\cdots-k ; 1}(x)=\frac{x}{U_{k-1}^{2}\left(\frac{1}{2 x}\right)}$;

(2) $D D_{1-2-\cdots-k ; 1}(x)=\frac{1}{U_{k-1}\left(\frac{1}{2 x}\right)} \sum_{m=0}^{k-2} \frac{x^{k-1-m}\left(x^{m+1}+U_{m-1}\left(\frac{1}{2 x}\right)\right)}{U_{m}\left(\frac{1}{2 x}\right) U_{m+1}\left(\frac{1}{2 x}\right)}$;

(3) $U U_{1-2-\cdots-k ; 1}(x)=\frac{1}{U_{k-1}^{2}\left(\frac{1}{2 x}\right)}$;

(4) $D U_{1-2-\cdots-k ; 1}(x)=\frac{1}{x U_{k-1}\left(\frac{1}{2 x}\right)} \sum_{m=0}^{k-2} \frac{x^{k-1-m}\left(x^{m+1}+U_{m-1}\left(\frac{1}{2 x}\right)\right)}{U_{m}\left(\frac{1}{2 x}\right) U_{m+1}\left(\frac{1}{2 x}\right)}$.

Proof. To verify (1), as consequence of Proposition 2.1, we have three possibilities for the block decomposition for an arbitrary up-down permutation $\pi=(\alpha, n, \beta) \in U D_{n}(1-3-2)$ that contain 1-2- $\cdots-k$ exactly once. The first possibility is $\alpha=(n-1)$ and $\beta \in U D_{n-2}(1-3-2)$ contains 1-2- - - $k$ exactly once. The second possibility is $\beta=(1)$ and $\alpha$ is up-down permutation of the numbers $2,3, \cdots, n-1$ which contains $1-2-\cdots-(k-1)$ exactly once. The third possibility satisfies the condition that every entry of $\alpha$ is greater than every entry of $\beta$, where $\alpha$ and $\beta$ are up-down permutations on the numbers $n-j+1, n-$ $j+2, \cdots, n-1$ and $1,2, \ldots, n-j$ (respectively) such that either $\alpha$ contains $1-2-\cdots-(k-1)$ exactly once and $\beta$ avoids 1-2- $\cdots-k$, or $\alpha$ avoids $1-2-\cdots-(k-1)$ and $\beta$ contains $1-2-\cdots-k$ exactly once. So, the first, the second, and the third cases above give contribution $x^{2} U D_{1-2-\cdots-k ; 1}(x), x^{2} U D_{1-2-\cdots-(k-1) ; 1}(x)$, $x U D_{1-2-\cdots-(k-1) ; 1}(x) U D_{1-2-\cdots-k ; 0}(x)+x U D_{1-2-\cdots-(k-1) ; 0}(x) U D_{1-2-\cdots-k ; 1}(x)$, respectively. Therefore,

$$
\begin{aligned}
& U D_{1-2-\cdots-k ; 1}(x)=x^{2} U D_{1-2-\cdots-k ; 1}(x)+x^{2} U D_{1-2-\cdots-(k-1) ; 1}(x)+ \\
& \quad+x U D_{1-2-\cdots-(k-1) ; 1}(x) U D_{1-2-\cdots-k ; 0}(x)+x U D_{1-2-\cdots-(k-1) ; 0}(x) U D_{1-2-\cdots-k ; 1}(x) .
\end{aligned}
$$


Using Theorem 2.4 together with Equation 1.3 and Recurrence 1.1, we get

$$
U D_{1-2-\cdots-k ; 1}(x)=x R_{k-1}^{2}\left(x^{2}\right) U D_{1-2-\cdots-(k-1) ; 1}(x) .
$$

Besides, by definitions we have that $U D_{1-2 ; 1}(x)=x^{3}$. Hence, by Identity 1.2 and by induction, case (1) holds.

Let us verify (2). Using the same arguments as in the proof of (1), we get

$$
\begin{array}{r}
D D_{1-2-\cdots-k ; 1}(x)=x U D_{1-2-\cdots-k ; 1}(x)+x D D_{1-2-\ldots-(k-1) ; 1}(x) U D_{1-2-\cdots-k ; 0}(x)+ \\
+x D D_{1-2-\ldots-(k-1) ; 0}(x) U D_{1-2-\cdots-k ; 1}(x)+x^{2} D D_{1-2-\cdots-(k-1) ; 1}(x),
\end{array}
$$

together with $D D_{1-2 ; 1}(x)=x^{4}$ (by the definitions). Hence, by using (1), Theorem 2.4 and induction on $k$, we get the desired result as claimed in $(2)$. The rest is easy to check.

As a corollary to Theorem 3.1 and Equation 1.4, we get the number of alternating permutations in $\mathfrak{S}_{n}(1-3-2)$ that contain $1-2-\cdots-k$ exactly once.

Corollary 3.2. For all $k \geq 3$,

$$
A_{1-2-\cdots-k ; 1}(x)=\frac{1+x}{U_{k-1}^{2}\left(\frac{1}{2 x}\right)} .
$$

For example, the number of alternating permutations in $A_{n}(1-3-2)$ that contain 1-2-3-4-5 exactly once is given by $\sum_{j=1}^{[n / 2]-3} F_{2 j} F_{2[n / 2]-4-2 j}$ for all $n \geq 6$, where $F_{m}$ is the $m$ th Fibonacci number.

3.2. A generalized patterns $\tau-3-\cdots-k$. In the current subsection we interesting in alternating permutations with two restrictions, the first one is 1-3-2 (classical pattern 132) and the second one is $\tau-3-\cdots-k$ (generalized pattern), where $\tau=12, \tau=21, \tau=1-2$, or $\tau=2-1$. Using the same arguments as in the proof of Theorem 3.1, we get

Theorem 3.3. Let $\tau \in\{12,21,1-2\}$. For all $k \geq 3$,

(1) $U D_{\tau-3-\cdots-k ; 1}(x)=\frac{x}{U_{k-1}^{2}\left(\frac{1}{2 x}\right)}$;

(2) $U U_{\tau-3-\cdots-k ; 1}(x)=\frac{1}{U_{k-1}^{2}\left(\frac{1}{2 x}\right)}$,

(3) $U D_{2-1-3 \cdots-k ; 1}(x)=U U_{2-1-3 \cdots-k ; 1}(x)=0$.

As a corollary to Theorem 3.3 and Equation 1.4 we get the number of alternating permutations in $A_{n}(1-3-2)$ that contain $\tau-3-\cdots-k$ exactly once, where $\tau \in\{12,21,1-2,2-1\}$.

Corollary 3.4. Let $\tau \in\{12,21,1-2\}$. For $k \geq 3$,

$$
A_{\tau-3-\cdots-k ; 1}(x)=\frac{(1+x) U_{k-2}\left(\frac{1}{2 x}\right)}{x U_{k-1}\left(\frac{1}{2 x}\right)}=(1+x) R_{k-1}\left(x^{2}\right) .
$$

Besides, $A_{2-1-3-\cdots-k ; 1}(x)=0$ for all $k \geq 3$.

Corollary 3.4 suggests that there should exist a bijection between the sets of alternating permutations of length $n$ that avoid 1-3-2 and contain 1-2- $\cdots-k$ exactly once and the set of alternating permutations of length $n$ that avoid 1-3-2 and contain 2-1-3- - - $-k$ exactly once. However, we failed to produce such a bijection, and finding it remains a challenging open question.

Again, by the same arguments as in the proof of Theorem 3.1, with using Proposition 2.1, we get the following result. 
Theorem 3.5. For all $k \geq 3$,

(1) $D D_{1-2-\cdots-k ; 1}(x)=\frac{x}{U_{k-1}\left(\frac{1}{2 x}\right)} \sum_{j=0}^{k-2} \frac{x^{k-2-j}\left(x^{j+1}+U_{j-1}\left(\frac{1}{2 x}\right)\right)}{U_{j}\left(\frac{1}{2 x}\right) U_{j+1}\left(\frac{1}{2 x}\right)}$,

(2) $D D_{12-3-4-\cdots-k ; 1}(x)=\frac{x}{U_{k-1}\left(\frac{1}{2 x}\right)}\left(x^{k}+\sum_{j=0}^{k-2} \frac{x^{k-2-j}\left(x^{j+1}+U_{j-1}\left(\frac{1}{2 x}\right)\right)}{U_{j}\left(\frac{1}{2 x}\right) U_{j+1}\left(\frac{1}{2 x}\right)}\right)$,

(3) $D D_{2-1-3-4-\cdots-k ; 1}(x)=\frac{x^{k-1}}{U_{k-1}\left(\frac{1}{2 x}\right)}$,

(4) $D D_{21-3-4-\cdots-k ; 1}(x)=\frac{1}{U_{k-1}^{2}\left(\frac{1}{2 x}\right)}$.

Moreover, for all $k \geq 3$,

$$
D U_{\tau-3-4-\cdots-k ; 1}(x)=\frac{1}{x} D D_{\tau-3-4-\cdots-k ; 1}(x),
$$

where $\tau \in\{12,21,1-2,2-1\}$

3.3. A three letter generalized pattern without dashes. In this subsection we find an explicit expression for $U D_{\tau ; r}(x), U U_{\tau ; r}(x), D U_{\tau ; r}(x), D D_{\tau ; r}(x)$, and $A_{\tau ; r}(x)$, where $\tau=123,213,231,312$, 321 are generalized pattern without dashes.

Theorem 3.6. For all $r \geq 1$,

(1) $U D_{123 ; r}(x)=U D_{321 ; r}(x)=0$,

(2) $U D_{213 ; r}(x)=\sum_{n \geq r+1} \frac{(r+1)}{n(n-r)}\left(\begin{array}{c}n \\ r+1\end{array}\right)^{2} x^{2 n+1}$,

(3) $U D_{231 ; r}(x)=\frac{1}{r+1}\left(\begin{array}{c}2 r \\ r\end{array}\right) x^{2 r+1}$ for $r \geq 1$,

(4) $U D_{312 ; r}(x)=\sum_{n \geq r+1} \frac{(r+1)}{n(n-r)}\left(\begin{array}{c}n \\ r+1\end{array}\right)^{2} x^{2 n+1}$.

Proof. First of all, let us define $U D_{\tau}(x, q)=\sum_{r \geq 0} U D_{\tau ; r}(x) q^{r}$ (similarly, we define $U U_{\tau}(x, q)$, $D U_{\tau}(x, q)$, and $\left.D D_{\tau}(x, q)\right)$.

The first case (1) holds by the definitions. To verify (2), let us use the same arguments as in the proof of Theorem 2.4. So, we have

$$
U D_{213}(x, q)=x^{2}\left(x+U D_{213}(x, q)\right)+x q U D_{213}(x, q)\left(x+U D_{213}(x, q)\right),
$$

equivalently,

$$
U D_{213}(x, q)=\frac{1-x^{2}(1+q)-\sqrt{x^{4}(1-q)^{2}-2 x^{2}(q+1)+1}}{2 x q} .
$$

The rest is easy to check.

Similarly, we have that

$$
U D_{231}(x, q)=x^{2} q\left(x+U D_{231}(x, q)\right)+x q U D_{231}(x, q)\left(x+U D_{231}(x)\right),
$$

equivalently,

$$
U D_{231}(x, q)=\frac{x^{3} q}{1-2 x^{2} q-x q U D_{231}(x, q)} .
$$

The rest is easy to check.

Using the arguments in the proof of the case (2) we get

$$
U D_{312}(x, q)=\frac{1-x^{2}(1+q)-\sqrt{x^{4}(1-q)^{2}-2 x^{2}(q+1)+1}}{2 x q} .
$$


Similarly to Theorem 3.6 we get the following result.

Theorem 3.7. Let $r \geq 0$.

(1) $U U_{123}(x ; r)=U U_{321}(x ; r)=0$;

(2) $U U_{213}(x ; r)=\sum_{n \geq r+1} \frac{(r+1)}{n(n-r)}\left(\begin{array}{c}n \\ r+1\end{array}\right)^{2} x^{2 n}$;

(3) $U D_{231}(x ; r)=\frac{1}{r+1}\left(\begin{array}{c}2 r \\ r\end{array}\right) x^{2 r+2}$ for $r \geq 1$;

(4) $U U_{312}(x ; r)=\sum_{n \geq r+1} \frac{(r+1)}{n(n-r)}\left(\begin{array}{c}n \\ r+1\end{array}\right)^{2} x^{2 n}$.

As a corollary to Theorem 3.6 and Theorem 3.7, we have the following result.

Theorem 3.8. Let $r \geq 0$.

(1) $A_{123}(x ; r)=A_{321}(x ; r)=0$;

(2) $A_{213}(x ; r)=A_{312}(x ; r)=\sum_{n \geq r+1} \frac{(r+1)}{n(n-r)}\left(\begin{array}{c}n \\ r+1\end{array}\right)^{2}\left(x^{2 n}+x^{2 n+1}\right)$;

(3) $A_{231}(x ; r)=C_{r}\left(x^{2 r+2}+x^{2 r+1}\right)$ for $r \geq 1$.

FiguRE 2. The second contribution for the block decomposition of a permutation that contain 1-3-2 exactly once

\section{Containing 1-3-2 eXACtly OnCE AND AVOIDing ARBitrary PATtern}

In this section we consider those alternating (up-up, up-down, down-up, down-down) permutations in $\mathfrak{S}_{n}$ that contain 1-3-2 exactly once and avoid an arbitrary generalized pattern $\tau$. We begin by setting some notation. Let $A_{\tau}^{1}(x)$ be the generating function for the number of alternating permutations in $A_{n}(\tau)$ that contain 1-3-2 exactly once. More precisely, We denote the generating function for number of up-up (resp. up-down, down-up, down-down) permutations in $\mathfrak{S}_{n}(\tau)$ that contain 1-3-2 exactly once by $U D_{\tau}^{1}(x)$ (resp. $U U_{\tau}^{1}(x), D U_{\tau}^{1}(x), D D_{\tau}^{1}(x)$ ). In this section we describe a method for enumerating alternating (up-up, up-down, down-up, down-down) permutations that contain 1-3-2 exactly once and avoid another generalized pattern. We begin with observation concerning the structure of the permutations that contain 1-3-2 exactly once.

Using the block decomposition approach (see [MV4]) we get two possibilities for the block decomposition for permutation $\pi$ containing 1-3-2 exactly once. The first one is $\alpha=\left(\alpha^{\prime}, n, \alpha^{\prime \prime}\right)$, where every entry of $\alpha^{\prime}$ is greater than every entry of $\alpha^{\prime \prime}$, and the second one is $\alpha=\left(\alpha^{\prime}, n-t+1, n, \alpha^{\prime \prime}, n-t+2, \alpha^{\prime \prime \prime}\right)$, where every entry of $\alpha^{\prime}$ is greater than $n-t+2, n-t+1$ is greater than every entry of $\alpha^{\prime \prime}$, and every entry of $\alpha^{\prime \prime}$ is greater than every entry of $\alpha^{\prime \prime \prime}$, as described in Figures 1 and 2 . Therefore, by using 
the block decomposition of $\alpha$, we get the following proposition which is the base for all the results in this section.

Proposition 4.1. Let $\alpha \in \mathfrak{S}_{n}$ contains 1-3-2 exactly once. Then the block decomposition of $\alpha$ can have one of the following three forms:

(i) there exists $t$ such that $\alpha=\left(\alpha^{\prime}, n, \alpha^{\prime \prime}\right)$, where $\alpha^{\prime}$ is a permutation of $n-1, n-2, \ldots, n-t+1$ which contains 1-3-2 exactly once, and $\alpha^{\prime \prime}$ is a permutation of $1,2, \ldots, n-t$ which avoids $1-3-2$;

(ii) there exists $t$ such that $\alpha=\left(\alpha^{\prime}, n, \alpha^{\prime \prime}\right)$, where $\alpha^{\prime}$ is a permutation of $n-1, n-2, \ldots, n-t+1$ which avoids 1-3-2, and $\alpha^{\prime \prime}$ is a permutation of $1,2, \ldots, n-t$ which contains 1-3-2 exactly once;

(iii) there exist $t, u$ such that $\alpha=\left(\alpha^{\prime}, n-t+1, n, \alpha^{\prime \prime}, n-t+2, \alpha^{\prime \prime \prime}\right)$, where $\alpha^{\prime}$ is a permutation of $n-1, n-2, \ldots, n-t+3$ which avoids $1-3-2, \alpha^{\prime \prime}$ is a permutation of $n-t, n-t-1, \ldots, n-u+1$ which avoids 1-3-2, and $\alpha^{\prime \prime \prime}$ is a permutation of $1,2, \ldots, n-u$ which avoids 1-3-2.

As a remark, using the proposition above for an arbitrary permutation $\alpha$ which contains 1-3-2 exactly once together with the definitions of up-up, up-down, down-up, down-down permutations we can present all the possibilities of the block decomposition for any arbitrary up-up, up-down, down-up, down-down permutation, respectively.

4.1. The pattern $\tau=\varnothing$. Using Proposition 4.1, we now enumerate those alternating (up-up, updown, down-up, down-down) permutations in $\mathfrak{S}_{n}$ that contain 1-3-2 exactly once.

Theorem 4.2. We have

(1) The generating function for the number of up-down permutations in $\mathfrak{S}_{n}$ containing 1-3-2 exactly once is given by

$$
U D_{\varnothing}^{1}(x)=\frac{x\left(1-\sqrt{1-4 x^{2}}\right)}{1-4 x^{2}+\sqrt{1-4 x^{2}}} .
$$

In other words, the number of up-down permutations in $\mathfrak{S}_{n}$ containing 1-3-2 exactly once is given by $\left(\begin{array}{c}n-1 \\ (n-3) / 2\end{array}\right)$,

(2) The generating function for the number of up-up permutations in $\mathfrak{S}_{n}$ containing 1-3-2 exactly once is given by

$$
U U_{\varnothing}^{1}(x)=\frac{x^{2}-1}{x^{2}}+\frac{1-3 x^{2}}{x^{2} \sqrt{1-4 x^{2}}} .
$$

In other words, the number of up-up permutations in $\mathfrak{S}_{n}$ containing 1-3-2 exactly once is given by $2\left(\begin{array}{c}n-1 \\ (n-4) / 2\end{array}\right)$,

(3) The generating function for the number of down-down permutations in $\mathfrak{S}_{n}$ containing 1-3-2 exactly once is given by

$$
D D_{\varnothing}^{1}(x)=\frac{x^{2}-1}{x^{2}}+\frac{1-3 x^{2}}{x^{2} \sqrt{1-4 x^{2}}} .
$$

In other words, the number of down-down permutations in $\mathfrak{S}_{n}$ containing 1-3-2 exactly once is given by $2\left(\begin{array}{c}n-1 \\ (n-4) / 2\end{array}\right)$,

(4) The generating function for the number of down-up permutations in $\mathfrak{S}_{n}$ containing 1-3-2 exactly once is given by

$$
D U_{\varnothing}^{1}(x)=\frac{2 x^{4}+4 x^{2}-3}{2 x^{3}}+\frac{3-10 x^{2}}{2 x^{3} \sqrt{1-4 x^{2}}} .
$$


In other words, the number of down-up permutations in $\mathfrak{S}_{n}$ containing 1-3-2 exactly once is given by $\frac{3}{2}\left(\begin{array}{c}n+3 \\ (n+3) / 2\end{array}\right)-5\left(\begin{array}{c}n+1 \\ (n+1) / 2\end{array}\right)$,

where $\left(\begin{array}{l}a \\ b\end{array}\right)$ is assumed to be 0 whenever $a<b, b<0$, or $b$ is a non-integer number.

Proof. To verify (1), let us consider the three possibilities of the block decompositions, as described in Proposition 4.1, for an arbitrary permutation $\pi \in \mathfrak{S}_{n}$ that contain 1-3-2 exactly. The first (or the second) contribution of the block decomposition above for up-down permutations gives

$$
x U D_{\varnothing}^{1}(x)\left(x+U D_{\varnothing}(x)\right),
$$

and the third contribution of the block decomposition above for up-down permutations gives

$$
x^{3}\left(U U_{\varnothing}(x)\left(x+U D_{\varnothing}(x)\right)^{2}+\left(x+U D_{\varnothing}(x)\right)^{2}+U U_{\varnothing}(x)+1\right) .
$$

Therefore, by using the above three contributions, we have

$$
U D_{\varnothing}^{1}(x)=2 x U D_{\varnothing}^{1}(x)\left(x+U D_{\varnothing}(x)\right)+x^{3}\left(U U_{\varnothing}(x)\left(x+U D_{\varnothing}(x)\right)^{2}+(x+U D(x))^{2}+U U_{\varnothing}(x)+1\right) .
$$

Hence, by solving the above equation together with Theorem 2.2, we get the claimed in (1).

Similarly, let us verify (2). The first contribution of the block decomposition for up-up permutations gives $x U D_{\varnothing}^{1}(x)\left(U U_{\varnothing}(x)+1\right)$, the second contribution of the block decomposition for up-up permutations gives $x\left(U D_{\varnothing}(x)+x\right) U U_{\varnothing}^{1}(x)$, and the third contribution of the block decomposition for up-up permutations gives $x^{3}\left(U U_{\varnothing}(x)+1\right)^{2}\left(U D_{\varnothing}(x)+x\right)$. Therefore, by using the three three contributions above, we get

$$
U U_{\varnothing}^{1}(x)=x U D_{\varnothing}^{1}(x)\left(U U_{\varnothing}(x)+1\right)+x\left(U D_{\varnothing}(x)+x\right) U U_{\varnothing}^{1}(x)+x^{3}\left(U U_{\varnothing}(x)+1\right)^{2}\left(U D_{\varnothing}(x)+x\right) .
$$

Hence, by using (1) together with Theorem 2.2 we get the claimed in (2).

Similarly to (1) and (2), we can verify cases (3) and (4).

As a corollary to Theorem 4.2 together with Identity 1.4 we have

Corollary 4.3. The number of alternating permutations in $A_{n}$ containing 1-3-2 exactly once is given by

$$
\left(\begin{array}{c}
n-1 \\
(n-3) / 2
\end{array}\right)+\left(\begin{array}{c}
n-1 \\
(n-4) / 2
\end{array}\right)
$$

where $\left(\begin{array}{l}a \\ b\end{array}\right)$ is assumed to be 0 whenever $a<b, b<0$, or $b$ is a non-integer number.

Using Proposition 4.1 for alternating permutations, we now enumerate various sets of alternating (upup, up-down, down-up, down-down) permutations in $\mathfrak{S}_{n}$ that contain 1-3-2 exactly once and avoid nonempty generalized pattern $\tau$.

\subsection{A classical pattern $\tau=1-2-\cdots-k$.}

Theorem 4.4. For all $k \geq 3$,

(1) $U D_{1-2-\cdots-k}^{1}(x)=\frac{1}{U_{k-1}^{2}\left(\frac{1}{2 x}\right)} \sum_{j=0}^{k-3} U_{j+1}\left(\frac{1}{2 x}\right) U_{j}\left(\frac{1}{2 x}\right)$;

(2) $U U_{1-2-\cdots-k}^{1}(x)=\frac{1}{x U_{k-1}^{2}\left(\frac{1}{2 x}\right)}\left(x U_{k-3}^{2}\left(\frac{1}{2 x}\right)+\sum_{j=0}^{k-4} U_{j}\left(\frac{1}{2 x}\right) U_{j+1}\left(\frac{1}{2 x}\right)\right)$; 
(3) The generating function $D D_{1-2-\cdots-k}^{1}(x)$ is given by

$$
\frac{1}{U_{k-1}\left(\frac{1}{2 x}\right)} \sum_{i=0}^{k-2} x^{i}\left[\frac{x^{k-1-i}+U_{k-3-i}\left(\frac{1}{2 x}\right)}{U_{k-1-i}\left(\frac{1}{2 x}\right) U_{k-2-i}\left(\frac{1}{2 x}\right)}\left(\sum_{j=0}^{k-2-i} U_{j}\left(\frac{1}{2 x}\right) U_{j+1}\left(\frac{1}{2 x}\right)\right)-x U_{k-2-i}\left(\frac{1}{2 x}\right)\right] \text {; }
$$

(4) The generating function $D U_{1-2-\cdots-k}^{1}(x)$ is given by

$$
\frac{x^{k-1}+U_{k-3}\left(\frac{1}{2 x}\right)}{U_{k-2}\left(\frac{1}{2 x}\right)} U U_{1-2-\cdots-k}^{1}(x)+\frac{U_{k-2}\left(\frac{1}{2 x}\right)}{U_{k-1}\left(\frac{1}{2 x}\right)} D D_{k-1}^{1}(x)+\frac{x U_{k-3}\left(\frac{1}{2 x}\right)\left(x^{k-2}+U_{k-4}\left(\frac{1}{2 x}\right)\right)}{U_{k-2}\left(\frac{1}{2 x}\right) U_{k-1}\left(\frac{1}{2 x}\right)} .
$$

Proof. Using the same arguments as in the proof of Theorem 4.2, we get the following. The first contribution of the block decomposition for up-down permutations gives

$$
x^{2} U D_{1-2-\cdots-k}^{1}(x)+x U D_{1-2-\cdots-(k-1)}^{1}(x)\left(x+U D_{1-2-\cdots-k}(x)\right),
$$

the second contribution of the block decomposition for up-down permutations gives

$$
x U D_{1-2-\cdots-(k-1)}(x) U D_{1-2-\cdots-k}^{1}(x),
$$

and the third contribution of the block decomposition for up-down permutations gives

$$
x^{3}\left(1+U U_{1-2-\cdots-(k-1)}(x)\right)\left(\left(x+U D_{1-2-\cdots-(k-1)}(x)\right)\left(x+U D_{1-2-\cdots-k}(x)\right)+1\right) .
$$

Therefore, by using the three contributions above together with Theorem 2.4 and Recurrence 1.1, we have

$$
U D_{1-2-\cdots-k}^{1}(x)=\frac{U_{k-2}\left(\frac{1}{2 x}\right) U_{k-3}\left(\frac{1}{2 x}\right)}{U_{k-1}^{2}\left(\frac{1}{2 x}\right)}+\frac{U_{k-2}^{2}\left(\frac{1}{2 x}\right)}{U_{k-1}^{2}\left(\frac{1}{2 x}\right)} U D_{1-2-\cdots-(k-1)}^{1}(x) .
$$

Hence, by induction on $k$ together with $U D_{1-2}^{1}(x)=0$ (by the definitions) we get the desired result as claimed in (1).

Similarly, let us verify (2). The first contribution of the block decomposition for up-up permutations gives

$$
x^{2} U U_{1-2-\cdots-k}^{1}(x)+x U D_{1-2-\cdots-(k-1)}^{1}(x)\left(1+U U_{1-2-\cdots-k}(x)\right),
$$

the second contribution of the block decomposition for up-up permutations gives

$$
x U D_{1-2-\cdots-(k-1)}(x) U U_{1-2-\cdots-k}^{1}(x),
$$

and the third contribution of the block decomposition for up-up permutations gives

$$
x^{3}\left(x+U D_{1-2-\cdots-(k-1)}(x)\right)\left(1+U U_{1-2-\cdots-(k-1)}(x)\right)\left(1+U U_{1-2-\cdots-k}(x)\right) .
$$

Therefore, by using the three contributions above together with case (1), Theorem 2.4 and Recurrence 1.1 , we get the desired result as claimed in (2).

Similarly, we have

$$
\begin{aligned}
D D_{1-2-\cdots-k}^{1}(x) & =x\left(1+D D_{1-2-\cdots-(k-1)}(x)\right) U D_{1-2-\cdots-k}^{1}(x)+x\left(x+U D_{1-2-\cdots-k}(x)\right) D D_{1-2-\cdots-(k-1)}^{1}(x) \\
& +x^{3}\left(x+U D_{1-2-\cdots-(k-1)}(x)\right)\left(1+\left(x+U D_{1-2-\cdots-(k-1)}(x)\right)\left(x+U D_{1-2-\cdots-k}(x)\right)\right), \\
& \\
D U_{1-2-\cdots-k}^{1}(x) & =x\left(1+D D_{1-2-\cdots-(k-1)}(x)\right) U U_{1-2-\cdots-k}^{1}(x)+x\left(1+U U_{1-2-\cdots-k}(x)\right) D D_{1-2-\cdots-(k-1)}^{1}(x) \\
& +x^{3}\left(x+D U_{1-2-\cdots-(k-1)}(x)\right)\left(x+U D_{1-2-\cdots-(k-1)}(x)\right)\left(1+U U_{1-2-\cdots-k}(x)\right) .
\end{aligned}
$$

Using Theorem 2.4, cases (1) and (2), and Recurrence 1.1, we get (3).

As a corollary to Theorem 4.4 together with using Identity 1.4, we have 
Corollary 4.5. For all $k \geq 3$, the generating function $A_{1-2-\cdots-k}^{1}(x)$ given by

$$
\frac{1}{x U_{k-1}^{2}\left(\frac{1}{2 x}\right)}\left[x U_{k-3}\left(\frac{1}{2 x}\right)\left(U_{k-3}\left(\frac{1}{2 x}\right)+U_{k-4}\left(\frac{1}{2 x}\right)\right)+(1+x) \sum_{m=0}^{k-4} U_{m+1}\left(\frac{1}{2 x}\right) U_{m}\left(\frac{1}{2 x}\right)\right] \text {. }
$$

4.3. A generalized patterns $\tau=12-3-\cdots-k$ or $\tau=21-3-\cdots-k$. Using the same arguments as in the proof of Theorem 4.4, we get

Theorem 4.6. For all $k \geq 3$,

$$
\begin{array}{ll}
U D_{12-3-\cdots-k}^{1}(x)=U D_{1-2-\cdots-k}^{1}(x), & U U_{12-3-\cdots-k}^{1}(x)=U U_{1-2-\cdots-k}^{1}(x) \\
D U_{12-3-\cdots-k}^{1}(x)=D U_{1-2-\cdots-k}^{1}(x), & D D_{12-3-\cdots-k}^{1}(x)=D D_{1-2-\cdots-k}^{1}(x) .
\end{array}
$$

Moreover, for all $k \geq 3$,

$$
A_{\tau-3-\cdots-k}^{1}(x)=A_{1-2-\cdots-k}^{1}(x) .
$$

4.4. A classical pattern $\tau=2-1-3-\cdots-k$. Using the same arguments as in the proof of Theorem 4.4, we get

Theorem 4.7. For all $k \geq 3$,

(1) $U D_{2-1-3-\cdots-k}^{1}(x)=\frac{1}{U_{k-1}^{2}\left(\frac{1}{2 x}\right)}\left(\sum_{j=0}^{k-3} U_{j+1}\left(\frac{1}{2 x}\right) U_{j}\left(\frac{1}{2 x}\right)-x\right) ;$
(2) $U U_{2-1-3-\cdots-k}^{1}(x)=\frac{1}{x U_{k-1}^{2}\left(\frac{1}{2 x}\right)}\left(x U_{k-4}\left(\frac{1}{2 x}\right) U_{k-2}\left(\frac{1}{2 x}\right)+\sum_{j=0}^{k-4} U_{j}\left(\frac{1}{2 x}\right) U_{j+1}\right)$.

\section{Containing 1-3-2 eXACTly once AND ANOther ARBitrary PATtern}

In this section we consider those alternating (up-up, up-down, down-up, down-down) permutations in $\mathfrak{S}_{n}$ that contain 1-3-2 exactly once and contain an arbitrary generalized pattern $\tau$. We begin by setting some notation. Let $A_{\tau ; r}^{1}(x)$ be the generating function for the number of alternating permutations in $A_{n}$ that contain 1-3-2 exactly once and contain $\tau$ exactly $r$ times. Moreover, We denote the generating function for number of up-up (resp. up-down, down-up, down-down) permutations in $\mathfrak{S}_{n}$ that contain 1-3-2 exactly once and contain $\tau$ exactly $r$ times by $U D_{\tau ; r}^{1}(x)\left(\right.$ resp. $\left.U U_{\tau ; r}^{1}(x), D U_{\tau ; r}^{1}(x), D D_{\tau ; r}^{1}(x)\right)$.

One can try to obtain results similar to Theorems 2.4, 3.1, and 4.2, but expressions involved become extremely cumbersome. So we just present the case of the up-down permutations in $\mathfrak{S}_{n}$ that contain 1-3-2 exactly once and contain $1-2-\cdots-k$ exactly once. Using Proposition 4.1 together with Theorems 2.4, 3.1, and 4.4, we get the following result.

Theorem 5.1. Let $k \geq 2$, the generating function $U D_{1-2-\cdots-k ; 1}^{1}(x)$ is given by

$$
\frac{1}{U_{k-1}^{2}\left(\frac{1}{2 x}\right)} \cdot \sum_{j=0}^{k-3}\left[\frac{U_{j+1}\left(\frac{1}{2 x}\right)\left(U_{j+1}\left(\frac{1}{2 x}\right)+x U_{j}\left(\frac{1}{2 x}\right)\right)+2 x \sum_{i=0}^{j-1} U_{i}\left(\frac{1}{2 x}\right) U_{i+1}\left(\frac{1}{2 x}\right)}{U_{j+1}\left(\frac{1}{2 x}\right) U_{j+2}\left(\frac{1}{2 x}\right)}\right] .
$$

As a remark, the generating functions $U U_{1-2-\cdots-k ; 1}^{1}(x), D U_{1-2-\cdots-k ; 1}^{1}(x)$, and $D D_{1-2-\cdots-k ; 1}^{1}(x)$ have more complicated expressions since the equations, which these generating function satisfied to, expressed 
in terms of $U D_{1-2-\cdots-d ; 1}^{1}(x)$. For example, $U U_{1-2-\cdots-k ; 1}^{1}(x)$ satisfies

$$
\begin{gathered}
U U_{1-2-\cdots-k ; 1}^{1}(x)=x U D_{1-2-\cdots-(k-1) ; 1}^{1}(x)\left(1+U U_{1-2-\cdots-k}(x)\right)+x U D_{1-2-\cdots-k}^{1}(x) U U_{1-2-\cdots-k ; 1}(x)+ \\
+x U D_{1-2-\cdots-(k-1) ; 1}(x) U U_{1-2-\cdots-k}^{1}(x)+x\left(x+U D_{1-2-\cdots-(k-1)}(x)\right) U U_{1-2-\cdots-k ; 1}^{1}(x)+ \\
+x^{3} U U_{1-2-\ldots-(k-1) ; 1}(x)\left(x+U D_{1-2-\cdots-(k-1)}(x)\right)\left(1+U U_{1-2-\cdots-k}(x)\right)+ \\
+x^{3}\left(1+U U_{1-2-\cdots-(k-1)}(x)\right) U D_{1-2-\cdots-(k-1) ; 1}(x)\left(1+U U_{1-2-\cdots-k}(x)\right)+ \\
+x^{3}\left(1+U U_{1-2-\cdots-(k-1)}(x)\right)\left(x+U D_{1-2-\cdots-(k-1)}(x)\right) U U_{1-2-\cdots-k ; 1}(x) .
\end{gathered}
$$

\section{Further Results}

Here we present two directions to generalize and to extend the results of the previous subsections.

6.1. Statistics on alternating permutation that avoid 1-3-2. The first direction is to find a statistics on alternating permutations in $A_{n}$ that avoid 1-3-2.

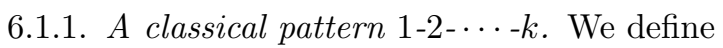

$$
\begin{aligned}
& U D_{1}\left(x_{1}, x_{2}, \ldots\right)=\sum_{n \geq 0} \sum_{\pi \in U D_{n}(1-3-2)} \prod_{j \geq 1} x_{j}^{1-2-\cdots-j(\pi)}, \\
& D U_{1}\left(x_{1}, x_{2}, \ldots\right)=\sum_{n \geq 0} \sum_{\pi \in D U_{n}(1-3-2)} \prod_{j \geq 1} x_{j}^{1-2-\cdots-j(\pi)}, \\
& U U_{1}\left(x_{1}, x_{2}, \ldots\right)=\sum_{n \geq 0} \sum_{\pi \in U U_{n}(1-3-2)} \prod_{j \geq 1} x_{j}^{1-2-\cdots-j(\pi)}, \\
& D D_{1}\left(x_{1}, x_{2}, \ldots\right)=\sum_{n \geq 0} \sum_{\pi \in D D_{n}(1-3-2)} \prod_{j \geq 1} x_{j}^{1-2-\cdots-j(\pi)},
\end{aligned}
$$

where $1-2-\ldots-j(\pi)$ is the number of occurrences of $1-2-\cdots-j$ in $\pi$.

Theorem 6.1. We have

(i) The generating function $U D_{1}\left(x_{1}, x_{2}, \ldots\right)$ is given by

$$
\frac{-\prod_{j \geq 1} x_{j}^{2\left({ }_{j-1}^{0}\right)}}{\prod_{j \geq 1} x_{j}^{\left(\begin{array}{c}
0 \\
j-1
\end{array}\right)}-\frac{1}{\prod_{j \geq 1} x_{j}^{\left({ }_{j-1}^{0}\right)}-\frac{1}{\prod_{j \geq 1} x_{j}^{2\left({ }_{j-1}\right)}}}}
$$

(ii) The generating function $U U_{1}\left(x_{1}, x_{2}, \ldots\right)$ is given by

$$
\frac{-1}{1-\frac{1}{x_{1}^{2}+x_{1} U D_{1}\left(x_{1} x_{2}, x_{2} x_{3}, \cdots\right)}} \text {. }
$$

(iii) The generating function $D D_{1}\left(x_{1}, x_{2}, \ldots\right)$ is given by

$$
\sum_{n \geq 1} \prod_{d=1}^{n} \frac{\prod_{j \geq 1} x_{j}^{2\left(\begin{array}{c}
d-1 \\
j-1
\end{array}\right)}}{1-\prod_{j \geq 1} x_{j}^{2\left(\begin{array}{c}
d-1 \\
j-1
\end{array}\right)}-\prod_{j \geq 1} x_{j}^{\left(\begin{array}{c}
d-1 \\
j-1
\end{array}\right)} U D_{1}\left(\prod_{j \geq 1} x_{j}^{\left(\begin{array}{c}
d \\
j
\end{array}\right)}, \prod_{j \geq 1} x_{j+1}^{\left(\begin{array}{c}
d \\
j
\end{array}\right)}, \ldots\right)} .
$$


(iv) The generating function $D U_{1}\left(x_{1}, x_{2}, \cdots\right)$ is given by

$$
x_{1} U U_{1}\left(x_{1}, x_{2}, \cdots\right)+x_{1} D D_{1}\left(x_{1} x_{2}, x_{2} x_{3}, \cdots\right)\left(1+U U_{1}\left(x_{1}, x_{2}, \cdots\right)\right) .
$$

Proof. Proposition 2.1(1) yields

$$
U D_{1}\left(x_{1}, x_{2}, \cdots\right)=x_{1}\left(U D_{1}\left(x_{1} x_{2}, x_{2} x_{3}, \cdots\right)+x_{1}\right)\left(U D_{1}\left(x_{1}, x_{2}, \cdots\right)+x_{1}\right),
$$

equivalently,

$$
U D_{1}\left(x_{1}, x_{2}, \cdots\right)=\frac{-x_{1}^{2}}{x_{1}-\frac{1}{x_{1}+U D_{1}\left(x_{1} x_{2}, x_{2} x_{3}, \cdots\right)}} .
$$

Hence, by induction, we get (i).

Proposition 2.1(3) yields

$$
U U_{1}\left(x_{1}, x_{2}, \cdots\right)=x_{1}\left(x_{1}+U D_{1}\left(x_{1} x_{2}, \cdots\right)\right)\left(1+U U_{1}\left(x_{1}, x_{2}, \cdots\right)\right),
$$

which equivalently to (ii).

Using Proposition 2.1(4), we get

$$
D D_{1}\left(x_{1}, x_{2}, \cdots\right)=x_{1}\left(x_{1}+U D_{1}\left(x_{1}, x_{2}, \cdots\right)\right)\left(1+D D_{1}\left(x_{1} x_{2}, x_{2} x_{3}, \cdots\right)\right),
$$

hence, by induction, we have (iii).

Finally, it is easy to check that Proposition 2.1(2) yields (iv).

We denote the generating function $C\left(x^{2}\right)-x=\frac{1-2 x^{2}-\sqrt{1-4 x^{2}}}{2 x}$ by $\widehat{C}(x)$. As an application to Theorem $6.1(\mathrm{i})$, we get the following:

(i) Let $x_{1}=x$ and $x_{j}=1$ for all $j \geq 2$. We have

$$
U D_{1}(x, 1,1, \ldots)=\sum_{n \geq 0} \sum_{\pi \in U} x_{D_{n}(1-3-2)}^{n}=\widehat{C}(x),
$$

as proved in Theorem 2.2.

(ii) Let $\pi$ be any permutation. We say that $\pi_{i}$ is right to left maximum if $\pi_{i}>\pi_{j}$ for all $j>i$. We denote the number of right to left maximum of $\pi$ by $\operatorname{rlmax}_{\pi}$. In [BCS], there were proved that $\operatorname{rlmax}_{\pi}=\sum_{j \geq 0}(-1)^{j+1} 1-2-\ldots-j(\pi)$. Therefore

$$
\sum_{n \geq 0} \sum_{\pi \in U} x^{n} y^{r \operatorname{lmax}}{ }_{D_{n}}=U D_{1}\left(x y, y^{-1}, y, y^{-1}, \ldots\right)=\frac{x^{2} y^{2}(x y+\widehat{C}(x))}{1-x^{2} y^{2}-x y \widehat{C}(x)} .
$$

In other words, the generating function for the number up-down permutations in $U D_{n}(1-3-2)$ having $k$ right to left maximum is given by

$$
x^{k} \sum_{j=0}^{k-2}\left(\begin{array}{c}
\frac{k-2+j}{2} \\
\frac{k-2-j}{2}
\end{array}\right) \widehat{C}^{j}(x) .
$$


(iii) Let $\pi$ be any permutations. The number of increasing subsequences in $\pi$ is given by $i n c_{\pi}=$ $\sum_{j \geq 0} 1-2-\cdots-j(\pi)$. Hence,

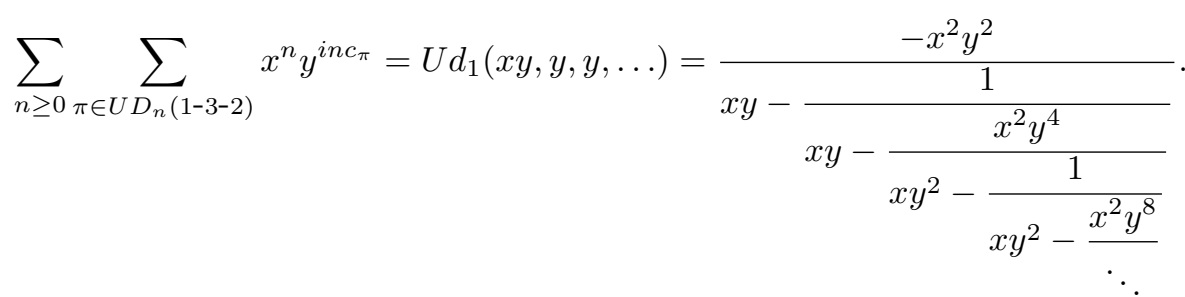

(iv) The generating function for the number of up-down permutations that avoid 1-3-2 and contain a prescribed number of occurrences of the pattern $1-2-\cdots-k$ is given by the continued fraction in the statement of Theorem 6.1 (i) together with $x_{1}=x, x_{k}=y$, and $x_{j}=1$ for all $j \neq 1, k$.

As an application to Theorem 6.1(ii), we have the following:

(i) The generating function for the number of up-up permutations in $\mathfrak{S}_{n}(1-3-2)$ is given by

$$
U U_{1}(x, 1,1, \ldots)=\frac{1-\sqrt{1-4 x^{2}}}{1+\sqrt{1-4 x^{2}}}
$$

(ii) The distribution for the number of right to left maximum of up-up permutations avoiding 1-3-2 is given by

$$
\begin{aligned}
& \sum_{n \geq 0} \sum_{\pi \in U U_{n}(1-3-2)} x^{|\pi|} y^{r l m a x} \max _{\pi}=U U_{1}\left(x y, y^{-1}, y, \ldots\right)= \\
& =\frac{-1}{1-\frac{1}{x^{2} y^{2}+x y \widehat{C}(x)}}=\sum_{d \geq 0} \sum_{j=0}^{d}\left(\begin{array}{c}
d-j \\
j
\end{array}\right) x^{2 j} \widehat{C}^{d-2 j}(x) y^{d} .
\end{aligned}
$$

(iii) The generating function for the number of up-up permutations that avoid 1-3-2 and contain a prescribed number of occurrences of the pattern $1-2-\cdots-k$ is given by the statement of Theorem 6.1 (ii) together with $x_{1}=x, x_{k}=y$, and $x_{j}=1$ for all $j \neq 1, k$.

As an application to Theorem 6.1(iii), we have the following:

(i) The generating function for the number of down-down permutations in $D D_{n}(1-3-2)$ is given by

$$
D D_{1}(x, 1,1, \ldots)=\frac{1-\sqrt{1-4 x^{2}}}{1+\sqrt{1-4 x^{2}}} .
$$

(ii) The distribution for the number of right to left maximum of $\pi \in D D_{n}(1-3-2)$ is given by

$$
\begin{gathered}
\sum_{n \geq 0} \sum_{\pi \in D D_{n}(1-3-2)} x^{|\pi|} y^{\operatorname{rlmax}_{\pi}}=D D_{1}\left(x y, y^{-1}, y, \ldots\right)=\frac{2 x^{2} y^{2}}{\sqrt{1-4 x^{2}}\left(1-x y \widehat{C}(x)-x^{2} y^{2}\right)}= \\
=\frac{2}{\sqrt{1-4 x^{2}}} \sum_{d \geq 2} \sum_{j=0}^{d-2}\left(\begin{array}{c}
d-2-j \\
j
\end{array}\right) \widehat{C}^{d-2 j}(x) x^{d} y^{d} .
\end{gathered}
$$

(iii) The generating function for the number of down-down permutations avoiding 1-3-2 and containing a prescribed number of occurrences of the pattern $1-2-\cdots-k$ is given by the statement of Theorem 6.1(iii) together with $x_{1}=x, x_{k}=y$, and $x_{j}=1$ for all $j \neq 1, k$. 
As an application to Theorem 6.1(iv), we have the following:

(i) The generating function for the number of down-up permutations in $D U_{n}(1-3-2)$ is given by

$$
D U_{1}(x, 1,1, \ldots)=x\left(\frac{1-\sqrt{1-4 x^{2}}}{2 x^{2}}\right)^{2}-x .
$$

(ii) The distribution for the number of right to left maximum of $\pi \in D U_{n}(1-3-2)$ is given by

$$
\begin{aligned}
& \sum_{n \geq 0} \sum_{\pi \in D U_{n}(1-3-2)} x^{|\pi|} y^{r \max \pi}=D U_{1}\left(x y, y^{-1}, y, \ldots\right)= \\
& =\frac{1-\sqrt{1-4 x^{2}}}{1+\sqrt{1-4 x^{2}}} x y+\frac{2}{\sqrt{1-4 x^{2}}} \sum_{d \geq 2} x^{d} y^{d} \sum_{j=0}^{d-1}\left({ }_{j}^{d-1-j}\right) \widehat{C}^{d-1-2 j}(x) .
\end{aligned}
$$

(iii) The generating function for the number of down-up permutations avoiding 1-3-2 and containing a prescribed number of occurrences of the pattern $1-2-\cdots-k$ is given by the statement of Theorem 6.1 (iv) together with $x_{1}=x, x_{k}=y$, and $x_{j}=1$ for all $j \neq 1, k$.

6.1.2. A generalized pattern $12-3-\cdots-k$. First of all, let us define

$$
\begin{aligned}
& U D_{2}\left(x_{1}, x_{2}, \ldots\right)=\sum_{n \geq 0} \sum_{\pi \in U D_{n}(1-3-2)} x_{1}^{|\pi|} \prod_{j \geq 2} x_{j}^{12-3-\cdots-j(\pi)}, \\
& U U_{2}\left(x_{1}, x_{2}, \ldots\right)=\sum_{n \geq 0} \sum_{\pi \in U U_{n}(1-3-2)} x_{1}^{|\pi|} \prod_{j \geq 2} x_{j}^{12-3-\cdots-j(\pi)},
\end{aligned}
$$

where $12-3-\cdots-j(\pi)$ is the number of occurrences of the generalized pattern $12-3-\ldots-j$ in $\pi$. Similarly to Theorem 6.1 we get the following result.

Theorem 6.2. We have

(i) The generating function $U D_{2}\left(x_{1}, x_{2}, \ldots\right)$ is given by

$$
\frac{-x_{1}^{2} \prod_{j \geq 2} x_{j}^{\left(\begin{array}{c}
0 \\
j-2
\end{array}\right)}}{x_{1} \prod_{j \geq 2} x_{j}^{\left({ }_{j-2}^{0}\right)}-\frac{1}{x_{1} \prod_{j \geq 2} x_{j}^{\left({ }_{j-2}^{1}\right)}-\frac{x_{1}^{2} \prod_{j \geq 2} x_{j}^{\left({ }_{j-2}^{1}\right)}}{x_{1}-\frac{1}{x_{1} \prod_{j \geq 2} x_{j}^{\left({ }_{j-2}^{2}\right)}-\frac{x_{1}^{2} \prod_{j \geq 2} x_{j}^{\left({ }_{j-2}^{2}\right)}}{x_{1}-} \cdot}}} ;}
$$

(ii) The generating function $U U_{2}\left(x_{1}, x_{2}, \ldots\right)$ is given by

$$
\frac{-1}{1-\frac{1}{x_{1}^{2} x_{2}+x_{1} x_{2} U D_{2}\left(x_{1}, x_{2} x_{3}, x_{3} x_{4}, \ldots\right)}} ;
$$

As an application to Theorem 6.2(i), we get that the number of up-down permutations avoiding 1-3-2 of length $2 n+1$ with $k$ rises is given by $C_{n} \delta_{k,(n-1) / 2}$, where $\delta_{a, b}=1$ if $a=b$, otherwise 0 .

As an another application, we get that the generating function for the number of up-down permutations avoiding 1-3-2 and containing a prescribed number of occurrences of the pattern 12-3-4- $\cdots-k$ is given by the statement of Theorem $6.2(\mathrm{i})$ together with $x_{1}=x, x_{k}=y$, and $x_{j}=1$ for all $j \neq 1, k$. For example, for $k=3$ we get 


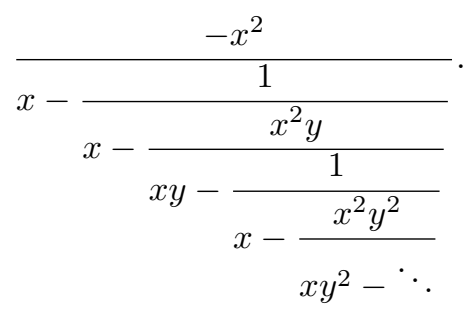

As an application to Theorem 6.2(ii), we get that the generating function for the number of upup permutations avoiding 1-3-2 and containing a prescribed number of occurrences of the pattern $12-3-4-\cdots-k$ is given by the statement of Theorem 6.2(ii) together with $x_{1}=x, x_{k}=y$, and $x_{j}=1$ for all $j \neq 1, k$. For example, for $k=3$ we get

$$
\frac{-1}{1-\frac{1}{x^{2}-x \cdot \frac{x^{2} y}{x y-\frac{1}{x-\frac{x^{2} y^{2}}{x y^{2}-} \cdot}}}} .
$$

As a corollary to Theorem $6.2(\mathrm{i}, \mathrm{ii})$, we have the following result.

Corollary 6.3. The generating function $\sum_{n \geq 0} \sum_{\pi \in A_{n}(1-3-2)} x_{1}^{|\pi|} \prod_{j \geq 2} x_{j}^{12-3-\cdots-j(\pi)}$ is given by

$$
1+x_{1}+\left(1+x_{1}\right) U U_{2}\left(x_{1}, x_{2}, x_{3}, \ldots\right) .
$$

Proof. Using Theorem 6.2(i,ii) we get that $U D_{2}\left(x_{1}, x_{2}, x_{3}, \cdots\right)=x_{1} U U_{2}\left(x_{1}, x_{2}, x_{3}, \cdots\right)$. On the other hand, by Identity 1.4 we have

$$
\sum_{n \geq 0} \sum_{\pi \in A_{n}(1-3-2)} x_{1}^{|\pi|} \prod_{j \geq 2} x_{j}^{12-3-\cdots-j}=1+x_{1}+U D_{2}\left(x_{1}, x_{2}, x_{3}, \cdots\right)+U U_{2}\left(x_{1}, x_{2}, x_{3}, \cdots\right) .
$$

Hence, by combining the two equations above we get the desired result.

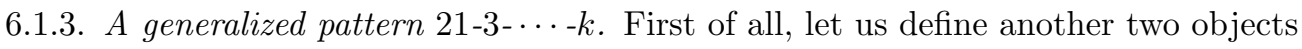

$$
\begin{aligned}
& U D_{3}\left(x_{1}, x_{2}, \ldots\right)=\sum_{n \geq 0} \sum_{\pi \in U D_{n}(1-3-2)} x_{1}^{|\pi|} \prod_{j \geq 2} x_{j}^{21-3-\cdots-j(\pi)}, \\
& U U_{3}\left(x_{1}, x_{2}, \ldots\right)=\sum_{n \geq 0} \sum_{\pi \in U U_{n}(1-3-2)} x_{1}^{|\pi|} \prod_{j \geq 2} x_{j}^{21-3-\cdots-j(\pi)},
\end{aligned}
$$

where $21-3-\cdots-j(\pi)$ is the number of occurrences of the generalized pattern $21-3-\ldots-j$ in $\pi$. Similarly to Theorem 6.1 we get

Theorem 6.4. We have

(i) $U D_{3}\left(x_{1}, x_{2}, \ldots\right)=U D_{2}\left(x_{1}, x_{2}, \ldots\right)$;

(ii) $U U_{3}\left(x_{1}, x_{2}, \ldots\right)=\frac{1}{x_{2}} U U_{2}\left(x_{1}, x_{2}, \ldots\right)$. 
Theorem 6.4 and Identity 1.4 together with the fact that $U D_{2}\left(x_{1}, x_{2}, x_{3}, \cdots\right)=x_{1} U U_{2}\left(x_{1}, x_{2}, x_{3}, \cdots\right)$ yield the following corollary.

Corollary 6.5. The generating function $\sum_{n \geq 0} \sum_{\pi \in A_{n}(1-3-2)} x_{1}^{|\pi|} \prod_{j \geq 2} x_{j}^{21-3-\cdots-j(\pi)}$ is given by

$$
1+x_{1}+\left(x_{1}+\frac{1}{x_{2}}\right) U U_{2}\left(x_{1}, x_{2}, x_{3}, \ldots\right) .
$$

6.2. Counting occurrences of 1-3-2 in an alternating permutation. The second direction is to find the generating function $A_{\tau}(x ; r)$ for the number of alternating permutation in $\mathfrak{S}_{n}$ that contain $\tau$ exactly $r$ times. Moreover, to find the generating functions $U U_{\tau}(x ; r), U D_{\tau}(x ; r), D U_{\tau}(x ; r)$, and $D D_{\tau}(x ; r)$ for the number of up-up, up-down, down-up, and down-down permutation in $\mathfrak{S}_{n}$ that contain $\tau$ exactly $r$ times, respectively.

For $\tau=1-3-2$, the block decomposition approach [MV5] in the alternating (up-up, up-down, down-up, down-down) permutations gives a complete answer for any given $r$ (as described in Section 2 for $r=0$, and Section 4 for $r=1$ ), and we get the following result.

Theorem 6.6. We have

(1)

$$
\left\{\begin{array}{l}
U D_{1-3-2}(x ; 0)=\frac{1-2 x^{2}}{2 x}-\frac{1}{2 x}\left(1-4 x^{2}\right)^{\frac{1}{2}} \\
U D_{1-3-2}(x ; 1)=\frac{-1}{2 x}+\frac{1-2 x^{2}}{2 x}\left(1-4 x^{2}\right)^{\frac{-1}{2}} \\
U D_{1-3-2}(x ; 2)=\frac{1}{2 x}-\frac{1-6 x^{2}+6 x^{4}}{2 x}\left(1-4 x^{2}\right)^{\frac{-3}{2}} \\
U D_{1-3-2}(x ; 3)=\frac{-2\left(1-x^{2}\right)}{x}+\frac{2-22 x^{2}+80 x^{4}-98 x^{6}+16 x^{8}}{x}\left(1-4 x^{2}\right)^{\frac{-5}{2}} \\
U U_{1-3-2}(x ; 0)=\frac{1-2 x^{2}}{2 x^{2}}-\frac{1}{2 x^{2}}\left(1-4 x^{2}\right)^{\frac{1}{2}} ; \\
U U_{1-3-2}(x ; 1)=\frac{x^{2}-1}{x^{2}}+\frac{1-3 x^{2}}{x^{2}}\left(1-4 x^{2}\right)^{\frac{-1}{2}} ; \\
U U_{1-3-2}(x ; 2)=\frac{4-5 x^{2}}{2 x^{2}}-\frac{4-29 x^{2}+54 x^{4}-16 x^{6}}{2 x^{2}}\left(1-4 x^{2}\right)^{\frac{-3}{2}} ; \\
U U_{1-3-2}(x ; 3)=\frac{13-11 x^{2}+2 x^{4}}{2 x^{2}}+\frac{13-152 x^{2}+612 x^{4}-940 x^{6}+384 x^{8}}{2 x^{2}}\left(1-4 x^{2}\right)^{\frac{-5}{2}}
\end{array}\right.
$$

$$
(3)\left\{\begin{array}{l}
A_{1-3-2}(x ; 0)=\frac{1+x-2 x^{2}-2 x^{3}}{2 x^{2}}-\frac{1+x}{2 x^{2}}\left(1-4 x^{2}\right)^{\frac{1}{2}} \\
A_{1-3-2}(x ; 1)=\frac{2 x^{2}-x-2}{2 x^{2}}+\frac{2+x-6 x^{2}-2 x^{3}}{2 x^{2}}\left(1-4 x^{2}\right)^{\frac{-1}{2}} \\
A_{1-3-2}(x ; 2)=\frac{4+x-5 x^{2}}{2 x^{2}}-\frac{4+x-29 x^{2}-6 x^{3}+54 x^{4}+6 x^{5}-16 x^{6}}{2 x^{2}}\left(1-4 x^{2}\right)^{\frac{-3}{2}} \\
A_{1-3-2}(x ; 3)=\frac{13-4 x-11 x^{2}+4 x^{3}+2 x^{4}}{2 x^{2}}+\frac{13+4 x-152 x^{2}-44 x^{3}+612 x^{4}+160 x^{5}-940 x^{6}-196 x^{7}+384 x^{8}+32 x^{9}}{2 x^{2}}\left(1-4 x^{2}\right)^{\frac{-5}{2}}
\end{array}\right.
$$

Finally, we conclude with some open problems suggested by the results in the previous sections. For example: (1) We found that the number of alternating permutations in $A_{n}(1-3-2,1-2-\cdots-k)$ is equal 
to the number of alternating permutations in $A_{n}(1-3-2,2-1-3-\cdots-k)$ (see Corollary 2.9). The question, if there is a bijective combinatorial proof for this property. (2) If there exists an explicit formula for $A_{1-3-2}(x ; r)$ for any given $r \geq 0$. (3) If there exist bijective combinatorial proofs for the formulas in the statement of Theorem 6.6.

Acknowledgments. The author is grateful to the anonymous referee for his helpful comments.

\section{REFERENCES}

[A1] D. André, Developments de $\sec x$ et $\tan x$, C.R. Acad. Sci., Paris 88 (1879), 965-967.

[A2] D. Andrś, memire sur les permutations alternées, J. Math. 7 (1881), 167-184.

[Bo] M. Bóna, The permutation classes equinumerous to the smooth class, Electron. J. Combin. 5 (1998), \#R31.

[BS] E. Babson and E. Steingrímsson, Generalized permutation patterns and a classification of the Mahonian statistics, Séminaire Lotharingien de Combinatoire, B44b:18pp, (2000).

[BCS] P. Brändén, A. Claesson, and E. Steingrímsson, Continued fractions and increasing subsequences in permutations, Discr. Math., to appear.

[C] A. Claesson, Generalised pattern avoidance, Europ. J. Combin. 22 (2001), 961-973.

[CW] T. Chow and J. West, Forbidden subsequences and Chebyshev polynomials, Discr. Math. 204 (1999), 119-128.

[Kn] D.E. Knuth, The Art of Computer Programming, 2nd ed. Addison Wesley, Reading, MA (1973).

[Km] D. Kremer, Permutations with forbidden subsequences and a generalized Schröder number, Discr. Math. 218 (2000), 121-130.

[Kr] C. Krattenthaler, Permutations with restricted patterns and Dyck paths, Adv. Appl. Math. 27 (2001), 510-530.

[M1] T. Mansour, Continued fractions and generalized patterns, Europ. J. Combin. 23:3 (2002), 329-344.

[M2] T. Mansour, Continued fractions, statistics, and generalized patterns, Ars Combinatorica, to appear (2002), preprint $\mathrm{CO} / 0110040$.

[M3] T. Mansour, Restricted 1-3-2 permutations and generalized patterns, Annals of Combinatorics 6 (2002), 65-76.

[MV1] T. Mansour and A. Vainshtein, Restricted permutations, continued fractions, and Chebyshev polynomials Electron. J. Combin. 7 (2000), \#R17.

[MV2] T. Mansour and A. Vainshtein, Restricted 132-avoiding permutations, Adv. Appl. Math. 126 (2001), 258-269.

[MV3] T. Mansour and A. Vainshtein, Layered restrictions and Chebychev polynomials, Annals of Combin. 5 (2001), 451-458.

[MV4] T. Mansour and A. Vainshtein, Restricted permutations and Chebyshev polynomials, Séminaire Lotharingien de Combinatoire 47 (2002), Article B47c.

[MV5] Counting occurrences of 132 in a permutation, Adv. Appl. Math. 28:2 (2002), 185-195.

[R] A. Robertson, Permutations containing and avoiding 123 and 132 patterns, Disc. Math. and Theo. Comp. Sci. 3 (1999), 151-154.

[Ri] Th. Rivlin, Chebyshev polynomials. From approximation theory to algebra and number theory, John Wiley, New York (1990).

[RWZ] A. Robertson, H. Wilf, and D. Zeilberger, Permutation patterns and continuous fractions, Elec. J. Combin. 6 (1999), \#R38.

[SP] N.J.A. Sloane and S. Plouffe, The Encyclopedia of Integer Sequences, Academic Press, New York (1995).

[SS] R. Simion, F.W. Schmidt, Restricted Permutations, Europ. J. Combin. 6 (1985), 383-406.

[W] J. West, Generating trees and forbidden subsequences, Discr. Math. 157 (1996), 363-372. 